\title{
The Benefits of Probiotics on Human Health
}

\section{Ivonne Figueroa-González', Alma Cruz-Guerrero ${ }^{1}$ and Guillermo Quijano ${ }^{2 *}$}

${ }^{1}$ Departamento de Biotecnología, Grupo de Biotecnología Alimentaria, Universidad Autónoma Metropolitana-Iztapalapa, Av. San Rafael Atlixco No. 186 Col. Vicentina, C.P. 09340. Distrito Federal, México.

${ }^{2}$ Department of Chemical Engineering and Environmental Technology, University of Valladolid, Dr. Mergelina s/n, 47011, Valladolid, Spain

\begin{abstract}
Nowadays it is well established that there is a strong relation between diet and health. The concept of using probiotic microorganisms to prevent and treat a variety of human diseases has been used for more than 100 years. Recently, there has been an increase in research of probiotics which has led to significant advances in our understanding of those microorganisms. The importance of probiotics is meaningful because have both, an application on industrial product development and a beneficial effect in human health. In this regard, probiotic microorganisms have been widely added to yogurts and other fermented milks, which are leader products of functional foods comprising approximately $65 \%$ of the world functional food market. The action mode of probiotic microorganisms is likely to be multifactorial and seems to be specific for each strain. By modifying the intestinal microbiota, probiotics directly or indirectly influence the state of health through providing end-products of anaerobic fermentation such as vitamins, short chain acids and bacteriocins giving protection against enteric microorganisms. Likewise, probiotic has influence on local and systemic immune responses, improve lactose intolerance symptoms, also stimulates toxin elimination and have other beneficial effects that are not mediated by the intestinal microbiota. In this work, the benefits provided to human health by probiotic microorganisms as well as the mechanisms potentially employed are reviewed and discussed.
\end{abstract}

Keywords: Biochemical mechanisms; Functional foods; Human health; Probiotics

\section{Introduction}

The original observation of the positive role of some bacteria can be credited to the pioneering work of Metcnikoff in the early 1900s. Almost a century ago, Metchnikoff noticed that Bulgarian peasants had an average life-span of 87 years, while approximately $4 \%$ of such population was able to reach 100 or more years of age. One of the major differences in their lifestyle in comparison with the contemporary diet was a large consumption of fermented milk [1]. This fact is related to the large consumption of bacteria contained in fermented milks used as a way to replacing harmful bacteria with useful ones. The term probiotic means "for life" and was created in the 1950s by Kollath [2], and was first coined by Lilly and Stillwell [3] for live bacteria and spores as animal feed supplements that should help limiting the use of antibiotics in animal husbandry. The first generally accepted definition was given by Fuller in 1992 [4]: a probiotic is "a live microbial feed supplement which beneficially affects the host by improving its intestinal microbiota balance". This definition was restricted to probiotics in animal nutrition. Actually, probiotics can defined as live microorganisms that, when administered in adequate amounts, confer a health benefit on the host $[5,6]$.

A growing attention in both basic research on the effect of probiotics in human health and the commercial advantages of the probiotic food concept has been observed in the last 30 years. Only 5 indexed publications with "probiotic" as topic were published in 1990, while more than 1000 publications were recorded by 2010 (Figure 1). This increased research has resulted in significant advances in the understanding of the fundamental mechanisms by which probiotics may confer beneficial effects to the host. Therefore, nowadays specific probiotic microorganisms are well characterized, allowing for the verification of their attributed health benefits $[6,7]$.

Probiotics can improve health benefits for conditions such as gastrointestinal infections and some functions of the digestive system, genitourinary infections, allergies and certain bowel disorders, all of which afflict a considerable proportion of the world population. However, considerable work is still needed to confirm these potential health benefits because the observed effects are usually related to

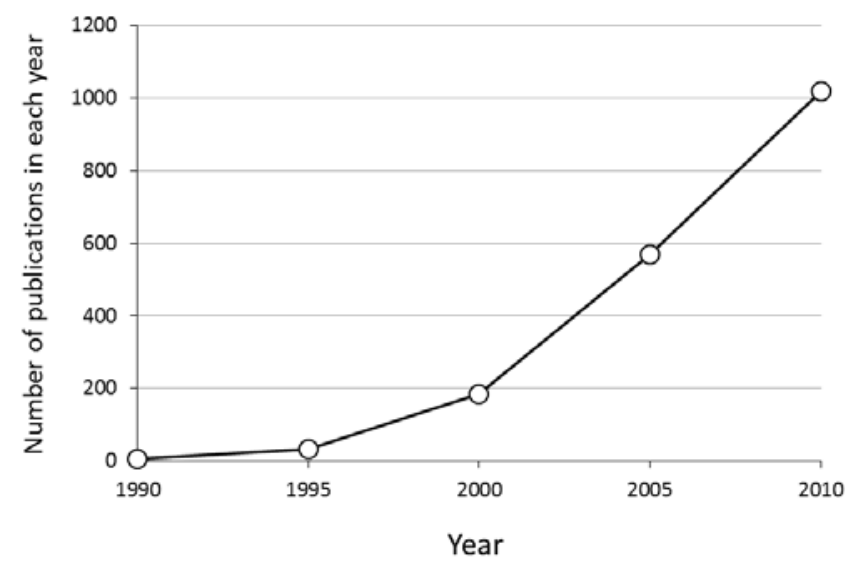

Figure 1: Evolution of the number of publications devoted to probiotics in the last 20 years. The data was obtained from ISI Web of Knowledge (Thompson Reuters, 2011) using "probiotic" as topic.

*Corresponding author: Ivonne Figueroa-González, Department of Chemical Engineering and Environmental Technology, University of Valladolid, Dr. Mergelina s/n, 47011, Valladolid, Spain, Tel: +34-983186424; Fax: +34-983423013; E-mail: gquijano@iq.uva.es

Received August 05, 2011; Accepted October 17, 2011; Published October 22 2011

Citation: Figueroa-González I, Cruz-Guerrero A, Quijano G (2011) The Benefits of Probiotics on Human Health. J Microbial Biochem Technol S1:003. doi:10.4172/1948-5948.S1-003

Copyright: () 2011 Figueroa-González I, et al. This is an open-access article distributed under the terms of the Creative Commons Attribution License, which permits unrestricted use, distribution, and reproduction in any medium, provided the original author and source are credited 
the presence of probiotics in the intestinal tract, ensured only by protracted administration. Long-term studies should complement the results already exists [8,9]. Likewise, probiotic food constitutes a sizeable part of the functional food market, and continues to grow at an exponential rate, with the potential for market growth estimated at a staggering US $\$ 120$ million per month [6]. The global market of probiotic ingredients, supplements and foods was worth $\$ 14.9$ billion in 2007 and it was expected to reach 15.9 billion in 2008, and 19.6 billion in 2013, representing a compound annual growth rate of 4.3\% [10]. The development of probiotic foods in the last two decades certainly represents an important advance in the food industry. This work summarizes the benefits provided to human health by probiotics as well as the fundamental mechanisms potentially employed by these microorganisms.

\section{Key characteristics of probiotic microorganisms}

Probiotics are defined as nonpathogenic microorganisms which, when administered in adequate amounts, confer a health benefit on the host and are able to prevent or improve some diseases [11]. Most of probiotic microorganisms belong to the genera Lactobacillus and Bifidobacterium, however, other bacteria and some yeast may have probiotic properties (Table 1). Lactobacilli are usually described as Gram-positive, non-spore-forming and non-flagelated rods or cocobacilli, aerotolerant, fastidious, acid-tolerant, and strictly fermentative. Glucose is fermented predominantly to lactic acid in the homofermentative case, or equimolar amounts of lactic acid, $\mathrm{CO}_{2}$ and ethanol (and or acetic acid) in the heterofermentative counterpart $[1,10]$. Lactobacilli have complex growth requirements (low oxygen tension, protein and its breakdown products, vitamins) and growth may occur in a temperature range between 30 and $45^{\circ} \mathrm{C}$. They are distributed in various ecological niches throughout the gastrointestinal and genital tracts and constitute an important part of indigenous microbiota of man and higher animals. On the other hand, Bifidobacteria are generally characterized as a Gram-positive, rod-shaped, non-gas-producing, non-spore forming, catalase negative and anaerobic microorganisms. They are saccharolytic organisms that produce acetic and lactic acid without generation of $\mathrm{CO}_{2}$. Bifidobacteria are microorganisms of paramount importance in the active and complex ecosystem of the intestinal tract of humans, the number of bifidobacteria decreasing with increasing the age of the host $[10,14]$.

Although the term probiotic is more related to lactic acid bacteria as Lactobacillus and Bifidobacterium, it can be extended to other microorganisms which have not been explored completely [10]. The probiotic effects of the microorganisms included in Table 1 have been well demonstrated in clinical trials. Other strains as Escherichia coli Nissle 1917 and Saccharomyces boulardii were considered as probiotics. However, genera such as Enterococcus and Bacillus despite being labeled as probiotics, they must be carefully considered since

\begin{tabular}{|l|l|l|}
\hline Lactobacilli & Bifidobacteria & Others \\
\hline L. casei Shirota & B. longum & Escherichia coli Nissle \\
L. rhamnosus GG & B. bifidum & Saccharomyces boulardii \\
L. johnsonii & B. infantis & Enterococcus faecalis \\
L. acidophilus & B. lactis & Lactococcus lactis \\
L. gasseri & B. breve & Propionibacteria \\
L. reuteri & B. animalis & \\
L. casei & B, adolescentis & \\
L. fermentum & & \\
L. crispatus & & \\
\hline
\end{tabular}

Table 1: Microorganisms commonly used as probiotics. Data from $[6,12,13]$. concerns still remain regarding the safe use of these organisms as probiotics [6]. Any microorganisms classified as probiotic has to prove efficacy and safety under recommended conditions for use in a defined population, methods of application and dosage. According to Kolida et al. [15], Mercenier et al. [18] and Fric [11] the microorganisms used as probiotics have to fulfill the following requirements: 1) detailed definition and typing, 2) absence of any pathogenic characteristics (including production of enterotoxins and cytotoxins, pathogenic adhesion, hemolysis, serum resistance, presence of genes of antibiotic resistance), 3) resistance to gastric acid and to bile, 4) clinically proven beneficial health-effects, 5) ability to adherence to the intestinal epithelium and 6) safety (no negative effects on the health are promoted).

The expected characteristics of probiotic microorganisms may include besides the physiological, immunological and metabolic activity, several technological properties such as been genetically stable strains, have a desired viability during processing and storage, good sensory properties and have a large-scale production [1].

\section{Commercially important probiotics}

The world population is becoming more conscious of the relation between nutrition and good health. This has stimulated research of the identification of food and food components that have special benefits in the consumer. In this context, functional foods based on probiotics like yogurts and fermented milks are nowadays leader products in the market [17]. Probiotic cultures have been exploited extensively by the dairy industry as a tool for the development of novel functional products. Traditionally, probiotics have been incorporated into yoghurt, milk and cheese; besides milk-based probiotic products, attempts are being made to use and develop non-milk probiotic products, a number of carriers for probiotics have been examined recently including edible spreads and meat [1]. Additionally, new products based on cereals, fruits and vegetables are in the development stages. Adding probiotics to different food is more complex than formulating dairy products because the bacteria need protection from the conditions of the new products and requires detailed knowledge of the functional characteristics of probiotics and the product development process [19]. Because of this, careful investigation of the interaction of different probiotics and food component should be considered in developing functional probiotic foods [20]. Probiotics are certainly very sensitive to many environmental stresses, such as acidity, oxygen and heat. The viability of probiotics is a key parameter to developing probiotic foods. Several factors (Figure 2) affect the viability of probiotic bacteria until they reach the target site of the host.

As noted by Figueroa-Gonzalez et al. [7], the commercial interest in functional foods containing probiotics matches with the increasing study of their role in the digestive tract. Fermented milks containing probiotics are result of the metabolism of lactic acid bacteria that grow in milk, its excellent nutritional quality being attributed mainly to the milk, which offers an important source of calcium, proteins and vitamins. The additional benefits on the health are result of the fermentation process, which enriches the fermented milk with a large number of microorganisms and fermentation products as organic acids and bioactive peptides. Therefore, fermented milk containing probiotic microorganisms provides a beneficial effect because their consumption takes to the ingestion of high amounts of live bacteria that exert benefits in the balance of the intestinal microbiota and health, beyond the basic nutrition [19]. Commercial cultures used in functional food market include mainly strains of Lactobacillus spp. and Bifidobacterium spp. 
Key cultures are included in Table 2.

\section{Beneficial effects of probiotics on human health}

The use of probiotics to prevent and treat a wide variety of conditions has gained favor in the past decade. This is, in part, due to a need to find alternatives to traditional therapies such as antibiotics as well as the lack of good treatments for gastrointestinal and other diseases. Consumption of probiotics is associated with a range of health benefits including stimulation of the immune system, protection against diarrheal diseases and nosocomial and respiratory tract infections, lowering of cholesterol, attenuation of overt immunoinflammatory disorders and anticancer effects [22,23]. It is important to note that health benefits provided by probiotics are strain specific, and not species- or genus-specific. Therefore, no probiotic strain will provide all proposed benefits, not even strains of the same species, and not all strains of the same species will be effective against defined healthy conditions [7]. A summary of the health effects related to probiotic ingestion is provided in Table 3. To the best of our knowledge, no risks on probiotic overdose in humans have been reported in the literature. According to Tijsseling et al. [30], most probiotic supplements contain approximately $1-5 \times 10^{9}$ viable bacteria per daily portion. This concentration compared with the number of bacteria in the intestines $\left(10^{14}\right)$ suggests that there is no overdose risk. However, potential risks cannot be ruled out.

\section{Mechanisms of probiotic activity}

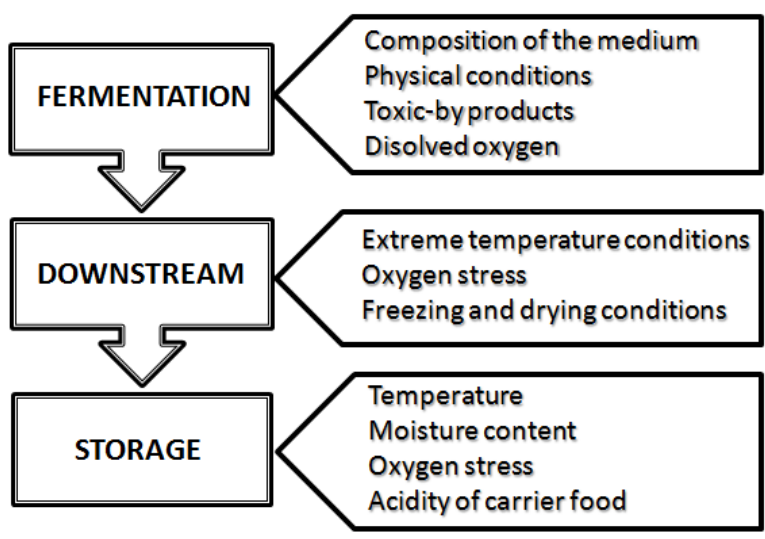

Figure 2: Factors influencing the stability of probiotics during functional foods production. Adapted from [21].

\begin{tabular}{|l|l|}
\hline Strain & Producer \\
\hline Lactobacillus casei Shirota & Yakult, Japan \\
Bifidobacterium lactis HN019 & Danisco, France \\
Lactobacillus rhamnosus GG & Valio, Finland \\
Lactobacillus johnsonii LA1 (NCC 533) & Nestlé, Switzerland \\
Bifidobacterium animalis DN-173 010 & Danone, France \\
Lactobacillus acidophilus LA-5 & Chr. Hansen, USA \\
Lactobacillus acidophilus NCFM & Danisco, France \\
Lactococcus lactis L1A & Essum AB, Sweden \\
Bifidobacterium longum BB536 & Snow Brand Milk Products, Japan \\
Lactobacillus casei DN-114 001 & Danone, France \\
Lactobacillus paracasei CRL431 & Chr. Hansen, USA \\
Lactobacillus casei F19 & Arla Food, Denmark \\
Lactobacillus acidophilus 145 & Danlac, Canada \\
Bifidobacterium animalis spp. lactis Bb12 & Chr. Hansen, USA \\
\hline
\end{tabular}

Table 2: Probiotic strains used in commercial products. Data from $[1,16,19]$.
Recent research clearly shows that the action mode of probiotic strains is complex and multifactorial and, from the existing evidence, appears to be strain specific $[25,31]$. The action mode depends on the metabolic properties, the kind of surface molecules expressed and the components to be secreted which probiotic actions a certain probiotic strain might show [25,32]. The effects of probiotics may be classified in three modes of action: 1) probiotics might be able to modulate the host's defenses including the innate and the acquired immune system as well as the intestinal barrier. This action mode is most likely important for the prevention and therapy of infectious diseases but also for the treatment of (chronic) inflammation of the digestive tract; 2) probiotics can also have a direct effect on the other microorganisms, commensal and/or pathogenic ones. This principle is in many cases of importance for the prevention and therapy of infections and restoration of the microbial equilibrium in the gut; 3 ) Finally, probiotic effects may be based on actions affecting microbial products like toxins, host products as bile salts and food ingredients. Such actions may result in inactivation of toxins and detoxification of host and food components in the gut. All three modes of probiotic action are in all likelihood involved in infection defense, prevention of cancer and in stabilizing or reconstituting the physiological balance between the intestinal microbiota and its host [10,33]. Additionally, probiotics may produce antagonistic substances like organic acids, hydrogen peroxide and bacteriocins $[1,13,19]$. A general view of the primary mechanisms of beneficial effects mediated by probiotics is showed in Figure 3 .

Because of the most important scientific question regarding the use of probiotics is the identification of the biochemical mechanisms by which these microorganisms impact on specific diseases. Hence, the potential mechanisms involved in the treatment of the most important diseases where probiotic ingestion has a proven beneficial effect are described below.

\section{Lactose intolerance}

The most investigated health effect of probiotics is the enhancement of lactose digestion and the avoidance of intolerance symptoms in lactose malabsorbers, namely persons with an insufficient activity of the lactose-cleaving enzyme $\beta$-galactosidase in the small intestine. Fermented milk contains probiotics that survives the passage through the stomach, to be finally deposited in the small intestine to support lactose hydrolysis by its own enzyme [12,25]. Additionally, probiotic bacteria contain high levels of lactase, which is released within the intestinal lumen when these bacteria are lysed by bile secretions. Lactase then acts on the ingested lactose decreasing maldigestion symptoms [25].

\section{Antipathogenic activity}

Many probiotics have been shown to produce antipathogenic compounds ranging from small molecules to bioactive antimicrobial peptides [22]. The lowering of $\mathrm{pH}$ by acids like lactic and acetic has bactericidal and bacteriostatic effects [17]. The production of $\mathrm{H}_{2} \mathrm{O}_{2}$ by probiotics strains may result in an antimicrobial effect due to the oxidation of sulfhydryl groups causing denaturing of a number of enzymes, and from the peroxidation of membrane lipids thus increasing membrane permeability [35]. Probiotics may produce antimicrobial peptides and bacteriocins, the antimicrobial action of these compounds involves increased permeability of the cytoplasmic membrane of the target cells, which leads to the release of small cytoplasmic particles, depolarization of the membrane potential and eventually to cell death [36]. In adition to producing antimicrobial compounds that act 
Citation: Figueroa-González I, Cruz-Guerrero A, Quijano G (2011) The Benefits of Probiotics on Human Health. J Microbial Biochem Technol S1:003. doi:10.4172/1948-5948.S1-003

Page 4 of 6

\begin{tabular}{|c|c|c|c|}
\hline \multicolumn{2}{|l|}{ Microorganism } & \multirow{2}{*}{$\begin{array}{l}\text { Effects on human health } \\
\text { Reduces nasal symptom-medication. } \\
\text { Has the strongest human health efficacy with respect to management of lactose } \\
\text { malabsorption, rotaviral diarrhea, antibiotic-associated diarrhea and Clostridium difficile } \\
\text { diarrhea. }\end{array}$} & \multirow{2}{*}{$\begin{array}{l}\text { Reference } \\
{[12,23,24]}\end{array}$} \\
\hline Lactobacilli & Lactobacillus casei Shirota & & \\
\hline & $\begin{array}{l}\text { Lactobacillus rhamnosus } \\
\text { GG }\end{array}$ & $\begin{array}{l}\text { Decreased duration of erythromycin-induced diarrhea. } \\
\text { Downregulates the immunoinflammatory response in milk-hypertensive subjects. } \\
\text { Remediates the inflammatory conditions through modulation of gastrointestinal } \\
\text { microbiota. }\end{array}$ & {$[13,19,23,25]$} \\
\hline & Lactobacillus casei & $\begin{array}{l}\text { Reduces the severity and duration of diarrhea. } \\
\text { Stimulates the immune system of the gut, alleviate the symptoms of Crohn's disease } \\
\text { and possess strong antimicrobial properties. }\end{array}$ & {$[13,26]$} \\
\hline \multirow[t]{3}{*}{ Bifidobacteria } & Bifidobacterium breve & $\begin{array}{l}\text { Activates the humoral immune system by augmenting anti-rotavirus IgA production or } \\
\text { anti-influenza virus. } \\
\text { Adheres to human intestinal epithelial cells and inhibits enteropathogen-cell } \\
\text { interactions. }\end{array}$ & {$[13,14]$} \\
\hline & Bifidobacterium bifidum & $\begin{array}{l}\text { Compete successfully for space and nutrients against pathogenic or putrefactive } \\
\text { bacteria. } \\
\text { Reduces the incidence of diarrhea. } \\
\text { Increases antibody responses and seroconversion rates. } \\
\text { Secretes lactic and acetic acids and helps to inhibit the development of invasive } \\
\text { pathogens such as Escherichia coli and Candida spp. } \\
\text { Prevents infectious diarrhea through a small protective effect, seen as reduced } \\
\text { shedding of rotavirus. }\end{array}$ & {$[23,26,27]$} \\
\hline & Bifidobacterium animalis & $\begin{array}{l}\text { Normalizes the intestinal motility of obstipated subjects. } \\
\text { Reduces the risk of acute diarrhea in children and adults. }\end{array}$ & {$[1,12]$} \\
\hline Yeasts & $\begin{array}{l}\text { Saccharomyces } \\
\text { cerevisiae Boulardii }\end{array}$ & $\begin{array}{l}\text { Prevents traveler's diarrhea. } \\
\text { Prevents the development of colitis and enterocolitis of pathogenic origin. } \\
\text { Reduces the risk and duration of antibiotic-associated diarrhea. } \\
\text { Affects Clostridium difficile or antibiotic-associated diarrhea by altering the gut } \\
\text { microbiota in a healthy mode. }\end{array}$ & {$[11,25,28,29]$} \\
\hline
\end{tabular}

Table 3: Proven effects of probiotics on human health.

directly on pathogens, probiotics may stimulate host antimicrobial defense pathways. The intestinal tract has a number of mechanisms for resisting the effects of pathogens including the production of defensins that are cationic antimicrobial peptides that are produced in a number of cell types including Panetch cells in the crypts of the small intestine and intestinal epithelial cells. Probiotics may stimulate the synthesis of defensin expression or alternatively produce proteases that themselves activate defensins in the intestinal lumen [22].

\section{Immunomodulatory activity}

The innate immune system via toll-like receptors recognizes a large group of chemical structures in pathogens and lipoteichoic acids which enables them to recognize foreign objects which trigger a cascade of immunological defense mechanisms [1]. Probiotics can influence the immune system by products like metabolites, cell-wall components (peptidoglycanes, lipopolysaccharides) and DNA. Probiotic products are recognized by host cells sensitive for these because they are equipped with recognition receptors [32]. Probiotics shown to have immunomodulatory properties through the inhibition of bacterial translocation, stimulation of phagocytes/macrophages and natural killer cells, increased proliferation in organs of the immune system (Peyer's patches spleen) and increased release of cytokines [12].

\section{Hypocholesterolaemic effects}

The use of probiotics to reduce the risk of hypercholesteremia seems very attractive especially if consumed as a part of a normal daily nutrition [1]. The use of animals and humans models to evaluate the effects of probiotics on serum cholesterol has been emphasized over the years. Human studies have shown promising evidence that wellestablished probiotics possess beneficial effects. Studies examining the efficacy of probiotics in reducing cholesterol often do not sufficiently address the mechanisms by which probiotics modulate hypocholesterolemic effects and the optimum dose, frequency, and duration of treatment for different probiotic stains. Several mechanisms have been hypothesized, which include enzymatic deconjugation of bile acids by bile-salt hydrolase of probiotics, assimilation of cholesterol by probiotics, co-precipitation of cholesterol with deconjugated bile, incorporation of cholesterol into the cellular membranes of probiotics during growth and conversion of cholesterol into coprostanol [16,37]. Even so, in most of the studies conducted, the real factor responsible for a reduction in cholesterol levels remains unknown [38].

\section{Prevention of inflammatory bowel disease}

Inflammatory bowel disease comprises a spectrum of disorders characterized by inflammation, ulceration and abnormal narrowing of the gastrointestinal tract resulting in abdominal pain, diarrhea and gastrointestinal bleeding $[1,25]$. Probiotic administration, either through regulation of the inflammatory response, enhancing barrier function to prevent the invasion of tight junctions or modulation of gut microbiota composition and/or activity might bring about relief in IBD symptoms or maintain remission from symptoms [25,31].

\section{Cancer prevention}

Antigenotoxicity, antimutagenicity and anticarcinogenicity are important potential functional properties of probiotics [1]. Cancer preventing properties are ascribed to probiotic bacteria in fermented milk products. Several mechanisms have been suggested as a cause of these effects and have been investigated in vitro and in animal experiments: inhibition of tumor-growth and proliferation of tumor cells by glycopeptides and cytotoxic metabolites of lactobacilli, reduction of (pro) carcinogenic, mutagenic, and genotoxic substances and cancer promoting enzymes in the colon due to modifications of the gut microbiota, a decrease in $\mathrm{pH}$ and chemical modification [12]. 
A)

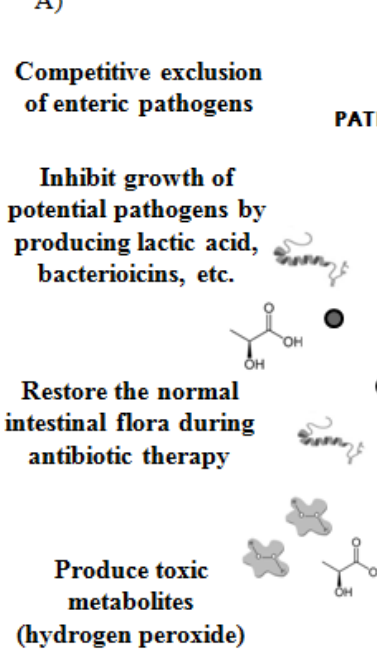

GUT LUMEN

B)

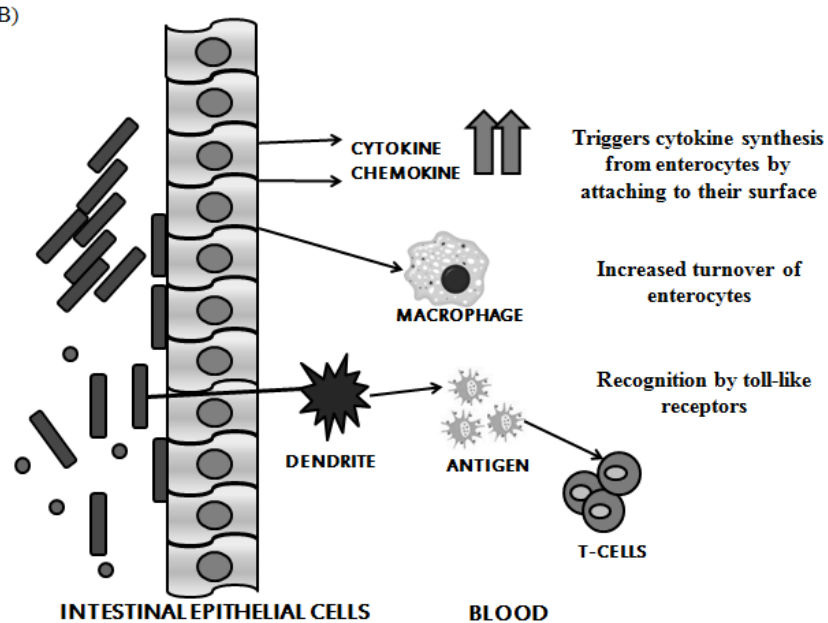

Figure 3: Proposed mechanisms employed by probiotic microorganisms to provide health benefits to the host: A) direct effect over commensal and pathogenic microorganisms; B) modulation of the host's defenses including the innate as well as the acquired immune system. Adapted from $[1,13,19,34]$

It is important to mention that the beneficial effects of probiotics related to intestinal tract could differ from one individual to another due to the difference of intestinal microbiota. This is because of probiotics, in general, should compete with indigenous bacteria for nutrients and niche in the intestine, but they may establish a symbiotic relationship with certain indigenous bacteria. Thus, the difference in indigenous intestinal microbiota significantly influences the magnitude of the probiotic effects. Therefore is quite likely that a probiotic strain that is effective for a particular animal species will not be suitable to other host species [39].

\section{Conclusion}

The growing attention in both basic research and commercial importance of probiotics is highlighted in this work. The tremendous increase in the number of scientific publications devoted to probiotics in the past 30 years resulted in: (i) important advances in the understanding of the fundamental mechanisms by which probiotics may confer beneficial effects to the host, and (ii) the ability to verify the attributed health benefits of probiotics. This scientific basis also resulted in a well-established credibility on the beneficial effects of probiotics in the human health and hence, nowadays there is a growing trend of the market for functional food based on probiotics. On the other hand, the so far reported mechanisms underlying the beneficial effects of probiotics are herein summarized, prevention of pathogen colonization and the stimulating of the host immune response being apparently the most important mechanisms. Finally, the understanding of the action mode of probiotic microorganism may be a powerful tool to design new strategies for the prevention and treatment of specific human diseases.

\section{Acknowledgements}

This work was financially supported by Secretaría de Educación Publica (SEP) through the program PROMEP (UAM-PTC-226) and Universidad Autónoma Metropolitana-Iztapalapa.

\section{References}

1. Vasiljevic T, Shah NP (2008) Probiotics -From Metchnikoff to bioactives. Int Dairy J 18: 714-728.

2. Kollath W (1953) Nutrition and the tooth system; general review with specia reference to vitamins. Deut Zahnaerztl Z 8: 7-16.

3. Lilly DM, Stillwell RH (1965) Probiotics: Growth-promoting factors produced by microorganisms. Science 147: 747-748.

4. Fuller R (1992) History and development of probiotics. In Probiotics, the scientific basis. London, UK: Chapman \& Hall pp. 1-8.

5. FAO/WHO (2002) Guidelines for the evaluation of probiotics in food. Report of a joint $\mathrm{FAO} / \mathrm{WHO}$. London, Ont., Canada.

6. Senok CA, Ismaeel YA, Botta GA (2005) Probiotics: facts and myths. Clin Microbiol Infect 11: 958-966.

7. Figueroa-González I, Quijano G, Ramírez G, Cruz-Guerrero A (2011) Probiotics and Prebiotics -perspectives and challenges. J Sci Food Agric 91: 1341-1348.

8. Aureli P, Capurso L, Castellazzi A, Clerici M, Giovannini M, et al. (2011) Probiotics and health : An evidence-based review. Pharmacol Res 63: 369372.

9. Heller KJ (2001) Probiotic bacteria in fermented foods: product characteristics and starter organisms. Am J Clin Nutr 73: 374S-379S.

10. Soccol CR, Porto de Souza L, Rigon M, Pedroni A, Tiemi C, et al. (2010) The potential of probiotics: A review. Food Technol Biotechnol 48: 413-134.

11. Fric $P$ (2007) Probiotics and prebiotics - renaissance of a therapeutic principle. Cent Eur J Med 2: 237-270.

12. de Vrese M, Schrezenmeir J (2008) Probiotics, prebiotics, and synbiotics. Adv Biochem Engin/Biotechnol 111: 1-66.

13. Kaur PI, Chopra K, Saini A (2002) Probiotics: potential pharmaceutical applications. Eur J Pharm Sci 15: 1-9.

14. Gomes A, Malcata FX (1999) Bifidobacterium spp. and Lactobacillus acidophilus: biological, biochemical, technological and therapeutical properties relevant for use as probiotics. Trends in Food Sci Tech 10: 139-157.

15. Kolida S, Saulnier DM, Gibson GR (2006) Gastrointestinal Microflora: Probiotics. Adv Appl Microbiol 249: 187-212.

16. Mercenier A, Pavan S, Pot B (2003) Probiotics as biotherapeutic agents: Present knowledge and future prospects. Curr Pharm Design 8: 99-110.

17. Agrawal R (2005) Probiotics: An emerging food supplement with health benefits. Food Biotechnol 19: 227-246.

18. Mattila-Sandholm T, Myllärinen P, Crittenden R, Mogensen G, Fondén R, et al (2002) Technological challenges for future probiotic foods. Int Dairy J 12: 173 182. 
Citation: Figueroa-González I, Cruz-Guerrero A, Quijano G (2011) The Benefits of Probiotics on Human Health. J Microbial Biochem Technol S1:003. doi:10.4172/1948-5948.S1-003

Page 6 of 6

19. Prado FC, Parada JL, Pandey A, Soccol CR (2008) Trends in non-dairy probiotic beverages. Food Res Int 41: 111-123

20. Ranadheera RDCS, Baines SK, Adams MC (2010) Importance of food in probiotic efficacy. Food Res Int 43: 1-7.

21. Lacroix C, Yildrim S (2007) Fermentation technologies for the production of probiotics with high viability and functionality. Curr Opin Biotech 18: 176-183.

22. Britton R, Versalovic J (2008) Probiotics and Gastrointestinal Infections. Interdisciplinary Perspectives on Infect Dis 2008: 1-10.

23. Gill H, Prasad J (2008) Bioactive Components of milk: Probiotics, immunomodulation, and health benefits, in Advances in Experimental Medicine and Biology, ed by Bösze Z. Springer, New York USA, pp 423-454.

24. Shah NP (2007) Functional cultures and health benefits. Int Dairy J 17: 1262 1277.

25. Tuohy KM, Probert HM, Smejkal CW, Gibson GR (2003) Using probiotics and prebiotics to improve gut health. Drug Discov Today 8: 692-699.

26. Pariyaporn I, Khawla S, Robinson RK (2003) The potential therapeutic benefits of consuming health promoting fermented dairy products: a brief update. Int $J$ Dairy Technol 56: 203-210.

27. Russell DA, Ross RP, Fitzgerald GF, Stanton C (2011) Metabolic activities and probiotic potential of bifidobacteria. Int J Food Microbiol 149: 88-105.

28. Priebe MG, Vonk RJ, Sun X, He T, Harmsen HJM, et al. (2002) The physiology of colonic metabolism. Possibilities for interventions with pre- and probiotics. Eur J Nutr 41:1/2-1/10

29. Im E, Pothoulakis C (2010) Recent Advances in Saccharomyces boulardi research. Gastroen Clin Biol 34: S62-S70.
30. Tijsseling IA, Pekelharing PR, Rombouts FM (2005) Position paper on probiotics. Agro Food Industry Hi-Tech 16: 30-32.

31. Santosa S, Farnworth E, Jones P (2006) Probiotics and Their Potential Health Claims. Nutr Rev 64: 265-274.

32. Vanderpool C, Yan F, Polk DB (2008) Mechanisms of probiotic action fo therapeutic applications in inflammatory bowel diseases. Inflamm Bowel Dis 14: 1585-1596.

33. Oelschlaeger TA (2010) Mechanisms of probiotic actions -A review. International J Med Microbiol 300: 57-62.

34. Turpin W, Humblot C, Thomas M, Guyot JM (2010) Lactobacilli as multifaceted probiotics with poorly disclosed molecular mechanisms. Int J Food Microbio 143: 87-102.

35. Ammor S, Tauveron G, Dufour E, Chevallier I (2006) Antibacterial activity of lactic acid bacteria against spoilage and pathogenic bacteria isolated from the same meat small-scale facility. Screening and characterization of the antibacterial compounds. Food Control 17: 454-461.

36. Simova ED, Beshkova, DB, Dimitrov P (2009) Characterization and antimicrobial spectrum of bacteriocins produced by lactic acid bacteria isolated from traditional Bulgarian dairy products. J Appl Microbiol 106: 692-701.

37. Ooi LG, Liong MT (2010) Cholesterol-lowering effects of probiotics and probiotics: A review of in vivo and in vitro findings. Int J Mol Sci 11: 2499-2522.

38. Dicks LMT, Botes M (2009) Probiotic lactic acid bacteria in the gastro-intestina tract: health benefits, safety and mode of action. Beneficial Microbes 1: 11-29.

39. Ohashi Y, Ushida K (2009) Health-beneficial effects of probiotics: Its mode of action. Animal Sci J 80: 361-371.

This article was originally published in a special issue, Probiotics handled by Editor(s). Dr. Arunachalam Muthaiyan, University of Arkansas, USA 